\title{
Antitone Galois Connections and Formal Concepts
}

\author{
Jung Mi Ko ${ }^{1}$ and Yong Chan Kim ${ }^{2}$ \\ Department of Mathematics, Gangneung-Wonju University, Gangneung, 201-702, Korea
}

\begin{abstract}
In this paper, we investigate the properties of antitone Galois connection and formal concepts. Moreover, we show that order reverse generating maps induce formal, attribute oriented and object oriented concepts on a complete residuated lattice.
\end{abstract}

Key Words : Complete residuated lattices, order reverse generating maps, isotone (antitone) Galois connetion, formal (resp. attribute oriented, object oriented) concepts

\section{Introduction and preliminaries}

Formal concept analysis is an important mathematical tool for data analysis and knowledge processing [1-4,8,10]. A fuzzy context consists of $(X, Y, R)$ where $X$ is a set of objects, $Y$ is a set of attributes and $R$ is a relation between $X$ and $Y$. Bělohlávek [1-4] developed the notion of formal concepts with $R \in L^{X \times Y}$ on a complete residuated lattice $L$.

In this paper, we investigate the properties of antitone Galois connections. Using their properties, we define formal, attribute oriented and object oriented concepts on a complete residuated lattice. Moreover, we show that order reverse generating maps induce formal, attribute oriented and object oriented concepts on a complete residuated lattice.

Definition 1.1. [9] A triple $(L, \leq, \odot)$ is called a complete residuated lattice iff it satisfies the following conditions:

(L1) $L=(L, \leq, 1,0)$ is a complete lattice where 1 is the universal upper bound and 0 denotes the universal lower bound;

(L2) $(L, \odot, 1)$ is a commutative monoid;

(L3) $\odot$ is distributive over arbitrary joins, i.e.

$$
\left(\bigvee_{i \in \Gamma} a_{i}\right) \odot b=\bigvee_{i \in \Gamma}\left(a_{i} \odot b\right)
$$

Define an operation $\rightarrow$ as $a \rightarrow b=\bigvee\{c \in L \mid a \odot c \leq$ $b\}$, for each $a, b \in L$.

Remark 1.2. [9] (1) Each frame $(L, \leq, \wedge)$ is a complete residuated lattice.

Manuscript received Nov. 24, 2009; revised May. 7, 2010; Accepted May. 8, 2010.

This work was supported by sabbatical year research funds from Gangneung-Wonju National University in 2009.
(2) The unit interval with a left-continuous t-norm $t$, $([0,1], \leq, t)$, is a complete residuated lattice.

(3) Define a binary operation $\odot$ on $[0,1]$ by $x \odot y=$ $\max \{0, x+y-1\}$. Then $([0,1], \leq, \odot)$ is a complete residuated lattice.

Let $(L, \leq, \odot)$ be a complete residuated lattice. A order reversing map * $: L \rightarrow L$ defined by $a^{*}=a \rightarrow 0$ is called a strong negation if $a^{* *}=a$ for each $a \in L$.

In this paper, we assume $\left(L, \leq, \odot,^{*}\right)$ is a complete residuated lattice with a strong negation $*$.

Lemma 1.3. [9] For each $x, y, z, x_{i}, y_{i} \in L$, we have the following properties.

(1) If $y \leq z, x \odot y \leq x \odot z, x \rightarrow y \leq x \rightarrow z$ and $z \rightarrow x \leq y \rightarrow x$.

(2) $x \odot y \leq x \wedge y$.

(3) $x \rightarrow\left(\bigwedge_{i \in \Gamma} y_{i}\right)=\bigwedge_{i \in \Gamma}\left(x \rightarrow y_{i}\right)$.

(4) $\left(\bigvee_{i \in \Gamma} x_{i}\right) \rightarrow y=\bigwedge_{i \in \Gamma}\left(x_{i} \rightarrow y\right)$.

(5) $\bigwedge_{i \in \Gamma} y_{i}^{*}=\left(\bigvee_{i \in \Gamma} y_{i}\right)^{*}$ and $\bigvee_{i \in \Gamma} y_{i}^{*}=\left(\bigwedge_{i \in \Gamma} y_{i}\right)^{*}$.

(6) $(x \odot y) \rightarrow z=x \rightarrow(y \rightarrow z)=y \rightarrow(x \rightarrow z)$.

(7) $x \odot y=\left(x \rightarrow y^{*}\right)^{*}$ and $x \rightarrow y=y^{*} \rightarrow x^{*}$.

\section{Antitone Galois connections and formal concepts}

Definition 2.1. [5] Let $X$ and $Y$ be two sets. Let $\omega^{\rightarrow}, \phi^{\rightarrow}, \xi^{\rightarrow}: L^{X} \rightarrow L^{Y}$ and $\omega^{\leftarrow}, \phi^{\leftarrow}, \xi^{\leftarrow}: L^{Y} \rightarrow L^{X}$ be operators.

(1) The pair $\left(\omega^{\rightarrow}, \omega^{\leftarrow}\right)$ is called antitone Galois connection between $X$ and $Y$ if for $\mu \in L^{X}$ and $\rho \in L^{Y}$, $\rho \leq \omega^{\rightarrow}(\mu)$ iff $\mu \leq \omega^{\leftarrow}(\rho)$.

(2) The pair $\left(\phi^{\rightarrow}, \phi^{\leftarrow}\right)$ is called an isotone Galois connection between $X$ and $Y$ if for $\mu \in L^{X}$ and $\rho \in L^{Y}$, $\phi^{\rightarrow}(\mu) \leq \rho$ iff $\mu \leq \phi^{\leftarrow}(\rho)$. Moreover, the pair $\left(\xi^{\leftarrow}, \xi^{\rightarrow}\right)$ 
is called an isotone Galois connection between $X$ and $Y$ if for $\mu \in L^{X}$ and $\rho \in L^{Y}, \xi^{\leftarrow}(\rho) \leq \mu$ iff $\rho \leq \xi^{\rightarrow}(\mu)$.

Definition 2.2. Let $\omega \rightarrow, \phi \rightarrow, \xi \rightarrow: L^{X} \rightarrow L^{Y}$ and $\omega^{\leftarrow}, \phi^{\leftarrow}, \xi^{\leftarrow}: L^{Y} \rightarrow L^{X}$ be functions. A pair $(\mu, \rho) \in$ $L^{X} \times L^{Y}$ is called:

(1) a formal concept if $\rho=\omega^{\rightarrow}(\mu)$ and $\mu=\omega^{\leftarrow}(\rho)$ where $\left(\omega^{\rightarrow}, \omega^{\leftarrow}\right)$ is an antitone Galios connection,

(2) an attribute oriented concept if $\rho=\phi \rightarrow(\mu)$ and $\mu=\phi^{\leftarrow}(\rho)$ where $\left(\phi^{\rightarrow}, \phi^{\leftarrow}\right)$ is an isotone Galios connection,

(3) an object oriented concept if $\rho=\xi \rightarrow(\mu)$ and $\mu=\xi^{\leftarrow}(\rho)$ where $\left(\xi^{\leftarrow}, \xi^{\rightarrow}\right)$ is an isotone Galios connection.

Theorem 2.3. Let $\omega^{\rightarrow}: L^{X} \rightarrow L^{Y}$ and $\omega^{\leftarrow}: L^{Y} \rightarrow L^{X}$ be operators. Let $\left(\omega^{\rightarrow}, \omega^{\leftarrow}\right)$ be an antitone Galois connection between $X$ and $Y$. Then the following properties hold:

(1) For each $\mu \in L^{X}$ and $\mu \in L^{X}, \mu \leq \omega^{\leftarrow}(\omega \rightarrow(\mu))$ and $\rho \leq \omega^{\rightarrow}\left(\omega^{\leftarrow}(\rho)\right)$.

(2) If $\mu_{1} \leq \mu_{2}$, then $\omega \rightarrow\left(\mu_{1}\right) \geq \omega \rightarrow\left(\mu_{2}\right)$. Moreover, if $\rho_{1} \leq \rho_{2}$, then $\omega^{\leftarrow}\left(\rho_{1}\right) \geq \omega^{\leftarrow}\left(\rho_{2}\right)$.

(3) For each $\mu \in L^{X}$ and $\rho \in L^{Y}$, $\omega^{\leftarrow}\left(\omega^{\rightarrow}\left(\omega^{\leftarrow}(\rho)\right)\right)=\omega^{\leftarrow}(\rho)$ and $\omega^{\rightarrow}\left(\omega^{\leftarrow}\left(\omega^{\rightarrow}(\mu)\right)\right)=$ $\omega \rightarrow(\mu)$.

(4) For each $\mu_{i} \in L^{X}$ and $\rho_{j} \in L^{Y}, \omega \rightarrow\left(\bigvee_{i \in I} \mu_{i}\right)=$ $\bigwedge_{i \in I} \omega \rightarrow\left(\mu_{i}\right)$ and $\omega^{\leftarrow}\left(\bigvee_{j \in J} \rho_{j}\right)=\bigwedge_{j \in J} \omega^{\leftarrow}\left(\rho_{j}\right)$.

(5) If $\omega^{\leftarrow}\left(\omega^{\rightarrow}\left(\mu_{i}\right)\right)=\mu_{i}$, then $\omega^{\leftarrow}\left(\omega \rightarrow\left(\bigwedge_{i \in I} \mu_{i}\right)\right)=$ $\bigwedge_{i \in I} \mu_{i}$

(6) If $\left(\omega^{\rightarrow}\left(\omega^{\leftarrow}\left(\rho_{j}\right)\right)=\rho_{j}\right.$, then $\omega^{\rightarrow}\left(\omega^{\leftarrow}\left(\bigwedge_{j \in J} \rho_{j}\right)\right)=$ $\bigwedge_{j \in J} \rho_{j}$.

Proof. (1) Since $\omega^{\rightarrow}(\mu) \leq \omega^{\rightarrow}(\mu)$, we have $\mu \leq$ $\omega^{\leftarrow}\left(\omega^{\rightarrow}(\mu)\right)$. Since $\omega^{\leftarrow}(\rho) \leq \omega^{\leftarrow}(\rho)$, we have $\rho \leq$ $\omega \rightarrow\left(\omega^{\leftarrow}(\rho)\right)$.

(2) Since $\mu_{1} \leq \mu_{2} \leq \omega^{\leftarrow}\left(\omega^{\rightarrow}\left(\mu_{2}\right)\right), \omega^{\rightarrow}\left(\mu_{2}\right) \leq$ $\omega^{\rightarrow}\left(\mu_{1}\right)$. Since $\omega^{\rightarrow}\left(\omega^{\leftarrow}\left(\rho_{2}\right)\right) \geq \rho_{2} \geq \rho_{1}, \omega^{\leftarrow}\left(\rho_{1}\right) \geq$ $\omega^{\leftarrow}\left(\rho_{2}\right)$.

(3) It easily proved from (1) and (2).

(4) By (2), $\omega \rightarrow\left(\bigvee_{i \in I} \mu_{i}\right) \leq \bigwedge_{i \in I} \omega \rightarrow\left(\mu_{i}\right)$. Since $\omega^{\rightarrow}\left(\mu_{i}\right) \geq \bigwedge_{i \in I} \omega^{\rightarrow}\left(\mu_{i}\right)$ implies $\omega^{\leftarrow}\left(\bigwedge_{i \in I} \omega^{\rightarrow}\left(\mu_{i}\right)\right) \geq$ $\mu_{i}$, we have $\bigvee_{i \in I} \mu_{i} \leq \omega^{\leftarrow}\left(\bigwedge_{i \in I} \omega^{\rightarrow}\left(\mu_{i}\right)\right)$. Hence $\omega \rightarrow\left(\bigvee_{i \in I} \mu_{i}\right) \geq \bigwedge_{i \in I} \omega \rightarrow\left(\mu_{i}\right)$.

(5) By (1), $\omega^{\leftarrow}\left(\omega^{\rightarrow}\left(\bigwedge \mu_{i}\right)\right) \geq \bigwedge \mu_{i}$ and by (2),

$$
\bigwedge_{i \in I} \mu_{i}=\bigwedge_{i \in I} \omega^{\leftarrow}\left(\omega^{\rightarrow}\left(\mu_{i}\right)\right) \geq \omega^{\leftarrow}\left(\omega^{\rightarrow}\left(\bigwedge_{i \in I} \mu_{i}\right)\right) .
$$

Hence, $\omega^{\leftarrow}\left(\omega^{\rightarrow}\left(\bigwedge_{i \in I} \mu_{i}\right)\right)=\bigwedge_{i \in I} \mu_{i}$.

(6) It is similarly proved as (5).

Example 2.4. Let $X=\{a, b, c\}, Y=\{x, y, z, w\}$ and $L=\{0,1\}$ be sets. Define $\omega \rightarrow: P(X) \rightarrow P(Y)$ and $\omega^{\leftarrow}: P(Y) \rightarrow P(X)$ as

$$
\omega^{\rightarrow}(\emptyset)=Y, \omega^{\rightarrow}(\{a\})=\{x, y\}, \omega^{\rightarrow}(\{b\})=\{y, w\},
$$

$$
\begin{gathered}
\omega^{\rightarrow}(\{c\})=\{z, w\}, \omega^{\rightarrow}(\{a, b\})=\{y\}, \\
\omega^{\rightarrow}(\{b, c\})=\{w\}, \omega^{\rightarrow}(\{a, c\})=\omega^{\rightarrow}(X)=\emptyset . \\
\omega^{\leftarrow}(\emptyset)=X, \omega^{\leftarrow}(\{w\})=\{b, c\}, \\
\omega^{\leftarrow}(\{z\})=\{c\}=\omega^{\leftarrow}(\{z, w\}), \\
\omega^{\leftarrow}(\{y\})=\{a, b\}, \omega^{\leftarrow}(\{y, w\})=\{b\}=\omega^{\leftarrow}(\{x, y\}), \\
\omega^{\leftarrow}(\{x\})=\{a\}, \omega^{\leftarrow}(\{y, z\})=\omega^{\leftarrow}(\{y, z, w\})=\emptyset, \\
\omega^{\leftarrow}(\{x, w\})=\omega^{\leftarrow}(\{x, z\})=\omega^{\leftarrow}(\{x, z, w\})=\emptyset, \\
\omega^{\leftarrow}(\{x, y, w\})=\omega^{\leftarrow}(\{x, y, z\})=\omega^{\leftarrow}(Y)=\emptyset .
\end{gathered}
$$

Then $\left(\omega^{\rightarrow}, \omega^{\leftarrow}\right)$ is an antitone Galios connection. Thus, we obtain formal concepts

$$
\begin{gathered}
\{(\emptyset, Y),(\{a\},\{x, y\}),(\{b\},\{y, w\}),(\{c\},\{z, w\}) \\
(\{b, c\},\{w\}),(\{a, b\},\{y\}),(X, \emptyset)\}
\end{gathered}
$$

In general, $\{z, w\}=\omega^{\rightarrow}(\{a, c\} \cap\{b, c\}) \neq \omega^{\rightarrow}(\{a, c\}) \cup$ $\omega \rightarrow(\{b, c\})=\{w\}$.

Definition 2.5. An operator $\phi^{\rightarrow}: L^{X} \rightarrow L^{Y}$ is called a join-generating operator, denoted by $\phi \rightarrow \in J(X, Y)$, if $\phi \rightarrow\left(\bigvee_{i \in \Gamma} \lambda_{i}\right)=\bigvee_{i \in \Gamma} \phi \rightarrow\left(\lambda_{i}\right)$, for $\left\{\lambda_{i}\right\}_{i \in \Gamma} \subset L^{X}$.

An operator $\psi^{\rightarrow}: L^{X} \rightarrow L^{Y}$ is called a meetgenerating operator, denoted by $\psi^{\rightarrow} \in M(X, Y)$, if $\psi \rightarrow\left(\bigwedge_{i \in \Gamma} \lambda_{i}\right)=\bigwedge_{i \in \Gamma} \psi \rightarrow\left(\lambda_{i}\right)$, for $\left\{\lambda_{i}\right\}_{i \in \Gamma} \subset L^{X}$.

An operator $\omega^{\rightarrow}: L^{X} \rightarrow L^{Y}$ is called an order reverse-generating operator, denoted by $\omega^{\rightarrow} \in K(X, Y)$, if $\omega \rightarrow\left(\bigvee_{i \in \Gamma} \lambda_{i}\right)=\bigwedge_{i \in \Gamma} \omega \rightarrow\left(\lambda_{i}\right)$, for $\left\{\lambda_{i}\right\}_{i \in \Gamma} \subset L^{X}$.

Theorem 2.6. For $\omega \rightarrow \in K(X, Y)$, Define functions $\phi_{\omega}, \xi_{\omega}: L^{X} \rightarrow L^{Y}$ and $\omega^{\leftarrow}, \phi_{\omega}^{\leftarrow}, \xi_{\omega}^{\leftarrow}: L^{Y} \rightarrow L^{X}$ as follows: for all $\lambda \in L^{X}, \rho \in L^{Y}$,

$$
\begin{gathered}
\omega^{\leftarrow}(\rho)=\bigvee\left\{\lambda \in L^{X} \mid \omega^{\rightarrow}(\lambda) \geq \rho\right\} \\
\phi_{\omega}^{\rightarrow}(\mu)=\left(\omega^{\rightarrow}(\mu)\right)^{*}, \phi_{\omega}^{\leftarrow}(\rho)=\omega^{\leftarrow}\left(\rho^{*}\right) \\
\xi_{\omega}^{\leftarrow}(\rho)=\bigwedge\left\{\lambda \in L^{X} \mid \omega\left(\lambda^{*}\right) \geq \rho\right\}, \\
\xi_{\omega}^{\rightarrow}(\mu)=\bigvee\left\{\rho \in L^{Y} \mid \xi_{\omega}^{\leftarrow}(\rho) \leq \mu\right\}
\end{gathered}
$$

Then the following properties hold:

(1) $\omega^{\leftarrow} \in K(Y, X)$ with $\omega^{\rightarrow}(\lambda) \geq \rho \Leftrightarrow \omega^{\leftarrow}(\rho) \geq \lambda$ for all $\lambda \in L^{X}$ and $\rho \in L^{Y}$. Furthermore, $\omega \rightarrow(\alpha \odot \lambda) \leq$ $\alpha \rightarrow \omega^{\rightarrow}(\lambda)$ iff $\alpha \rightarrow \omega^{\leftarrow}(\rho) \leq \omega^{\leftarrow}(\alpha \odot \rho)$. Similarly, $\omega^{\leftarrow}(\alpha \odot \lambda) \leq \alpha \rightarrow \omega^{\leftarrow}(\lambda)$ iff $\alpha \rightarrow \omega^{\rightarrow}(\rho) \leq \omega^{\rightarrow}(\alpha \odot \rho)$.

(2) The pair $\left(\omega^{\rightarrow}, \omega^{\leftarrow}\right)$ is an antitone Galois connection and $\left(\omega^{\leftarrow}\left(\omega^{\rightarrow}(\lambda)\right), \omega^{\rightarrow}(\lambda)\right)$ for all $\lambda \in L^{X}$ are formal concepts.

(3) The pair $\left(\phi_{\omega}, \phi_{\omega}^{\leftarrow}\right)$ is an isotone Galois connection and $\left(\phi_{\omega}^{\leftarrow}\left(\phi_{\omega}(\lambda)\right), \phi_{\omega}(\lambda)\right)=\left(\omega^{\leftarrow}\left(\omega^{\rightarrow}(\lambda)\right), \omega^{\rightarrow}(\lambda)^{*}\right)$ for all $\lambda \in L^{X}$ are attribute oriented concepts.

(4) $\omega^{\rightarrow}(\alpha \odot \mu) \leq \alpha \rightarrow \omega^{\rightarrow}(\mu)$ iff $\alpha \odot \phi_{\omega}(\mu) \leq$ $\phi_{\omega}(\alpha \odot \mu)$ iff $\alpha \rightarrow \phi_{\omega}^{\leftarrow}(\rho) \leq \phi_{\omega}^{\leftarrow}(\alpha \rightarrow \rho)$ 
(5) $\xi_{\omega}^{\leftarrow}: L^{Y} \rightarrow L^{X}$ is a join-generating function such that $\xi_{\omega}^{\leftarrow}(\rho)=\left(\omega^{\leftarrow}(\rho)\right)^{*}$ and

$$
\omega^{\rightarrow}\left(\lambda^{*}\right) \geq \rho \Leftrightarrow \omega^{\leftarrow}(\rho) \geq \lambda^{*} \Leftrightarrow \xi_{\omega}^{\leftarrow}(\rho) \leq \lambda .
$$

Moreover, $\omega^{\rightarrow}(\alpha \odot \lambda) \geq \alpha \rightarrow \omega^{\rightarrow}(\lambda)$ iff $\alpha \odot \xi_{\omega}^{\leftarrow}(\rho) \leq$ $\xi_{\omega}^{\leftarrow}(\alpha \odot \rho)$.

(6) $\xi_{\omega}: L^{X} \rightarrow L^{Y}$ is a meet-generating function such that $\xi_{\omega}(\lambda)=\omega^{\rightarrow}\left(\lambda^{*}\right)$ and

$\omega^{\rightarrow}\left(\lambda^{*}\right) \geq \rho \Leftrightarrow \omega^{\leftarrow}(\rho) \geq \lambda^{*} \Leftrightarrow \xi_{\omega}^{\leftarrow}(\rho) \leq \lambda \Leftrightarrow \rho \leq \xi_{\omega}(\lambda)$.

Moreover, $\omega \rightarrow(\alpha \odot \lambda) \geq \alpha \rightarrow \omega^{\rightarrow}(\lambda)$ iff $\alpha \rightarrow \xi_{\omega}(\lambda) \leq$ $\xi_{\omega}(\alpha \rightarrow \lambda)$.

(7) The pair $\left(\xi_{\omega}^{\leftarrow}, \xi_{\omega}\right)$ is an isotone Galois connection and $\left(\xi_{\omega}^{\leftarrow}(\rho), \xi_{\omega}\left(\xi_{\omega}^{\leftarrow}(\rho)\right)\right)=\left(\omega^{\leftarrow}(\rho)^{*}, \omega^{\rightarrow}(\omega \rightarrow(\rho))\right)$ for all $\rho \in L^{Y}$ are object oriented concepts.

Proof. (1) Since $\omega^{\rightarrow} \in K(X, Y)$ and $\omega^{\leftarrow}(\rho)=\bigvee\{\lambda \in$ $\left.L^{X} \mid \omega \rightarrow(\lambda) \geq \rho\right\}$, we have

$$
\omega^{\rightarrow}(\lambda) \geq \rho \Leftrightarrow \omega^{\leftarrow}(\rho) \geq \lambda .
$$

Moreover, $\omega^{\leftarrow} \in K(Y, X)$ from

$$
\begin{aligned}
\bigwedge_{i \in \Gamma} \omega^{\leftarrow}\left(\rho_{i}\right) \geq \mu & \Leftrightarrow \omega^{\leftarrow}\left(\rho_{i}\right) \geq \mu, \quad \forall i \in \Gamma \\
& \Leftrightarrow \omega \rightarrow(\mu) \geq \rho_{i}, \quad \forall i \in \Gamma \\
& \Leftrightarrow \omega \rightarrow(\mu) \geq \bigvee_{i \in \Gamma} \rho_{i}, \\
& \Leftrightarrow \omega^{\leftarrow}\left(\bigvee_{i \in \Gamma} \rho_{i}\right) \geq \mu .
\end{aligned}
$$

Hence $\omega^{\leftarrow}\left(\bigvee_{i \in \Gamma} \rho_{i}\right)=\bigwedge_{i \in \Gamma} \omega^{\leftarrow}\left(\rho_{i}\right)$.

Let $\omega \rightarrow(\alpha \odot \lambda) \leq \alpha \rightarrow \omega^{\rightarrow}(\lambda)$. For $\mu \leq \alpha \rightarrow \omega^{\leftarrow}(\rho)$, $\mu \odot \alpha \leq \omega^{\leftarrow}(\rho)$ iff $\omega^{\rightarrow}(\mu \odot \alpha) \geq \rho$. Since $\omega \rightarrow(\alpha \odot \mu) \leq$ $\alpha \rightarrow \omega^{\rightarrow}(\mu), \alpha \rightarrow \omega^{\rightarrow}(\mu) \geq \rho$ iff $\omega \rightarrow(\mu) \geq \alpha \odot \rho$ iff $\omega^{\leftarrow}(\alpha \odot \rho) \geq \mu$. Hence $\alpha \rightarrow \omega^{\leftarrow}(\rho) \leq \omega^{\leftarrow}(\alpha \odot \rho)$. Conversely, it similarly proved.

(2) By (1), since $\left(\omega^{\rightarrow}, \omega^{\leftarrow}\right)$ is an antitone Galois connection, $\left(\omega^{\leftarrow}\left(\omega^{\rightarrow}(\lambda)\right), \omega^{\rightarrow}(\lambda)\right)$ are formal concepts from Theorem 2.3(3).

(3) It follows from $\phi_{\omega}(\lambda) \leq \rho$ iff $\left(\omega^{\rightarrow}(\lambda)\right)^{*} \leq \rho$ iff $\omega^{\rightarrow}(\lambda) \geq \rho^{*}$ iff $\omega^{\leftarrow}\left(\rho^{*}\right) \geq \lambda$ iff $\phi_{\omega}^{\leftarrow}(\rho) \leq \lambda$. Moreover, $\phi_{\omega}^{\vec{\omega}}\left(\phi_{\omega}^{\overleftarrow{\omega}}\left(\phi_{\omega}(\lambda)\right)\right)=\phi_{\omega}\left(\phi_{\omega}^{\overleftarrow{\omega}}\left((\omega \rightarrow(\lambda))^{*}\right)\right)=$ $\phi_{\omega}\left(\omega^{\leftarrow}(\omega \rightarrow(\lambda))\right)=\left(\omega^{\rightarrow}\left(\omega^{\leftarrow}\left(\omega^{\rightarrow}(\lambda)\right)\right)\right)^{*}=$ $\left.\left(\omega^{\rightarrow}(\lambda)\right)\right)^{*}=\phi_{\omega}(\lambda)$

(4) $\omega^{\rightarrow}(\alpha \odot \mu) \leq \alpha \rightarrow \omega^{\rightarrow}(\mu)$ iff $(\omega \rightarrow(\alpha \odot \mu))^{*} \geq$ $\left(\alpha \rightarrow \omega^{\rightarrow}(\mu)\right)^{*}$ iff $\alpha \odot \phi_{\omega}(\mu) \leq \phi_{\omega}(\alpha \odot \mu)$.

Let $\alpha \rightarrow \phi_{\omega}^{\overleftarrow{\omega}}(\rho) \leq \phi_{\omega}^{\overleftarrow{\omega}}(\alpha \rightarrow \rho)$. For $\phi_{\omega}(\alpha \odot \mu) \leq \rho$, $\alpha \odot \lambda \leq \phi_{\omega}^{\leftarrow}(\rho)$. Then $\lambda \leq \alpha \rightarrow \phi_{\omega}^{\overleftarrow{\omega}}(\rho) \leq \phi_{\omega}^{\leftarrow}(\alpha \rightarrow \rho)$ implies $\phi_{\omega}(\lambda) \leq \alpha \rightarrow \rho$. Hence $\alpha \odot \phi_{\omega}(\lambda) \leq \rho$. Thus, $\alpha \odot \phi_{\omega}(\lambda) \leq \phi_{\omega}(\alpha \odot \lambda)$. Conversely, it is similarly proved.

(5) We have

$$
\begin{aligned}
& \xi_{\omega}^{\leftarrow}(\rho)=\bigwedge\left\{\lambda \in L^{X} \mid \omega^{\rightarrow}\left(\lambda^{*}\right) \geq \rho\right\} \\
& =\left(\bigvee\left\{\lambda^{*} \in L^{X} \mid \omega^{\rightarrow}\left(\lambda^{*}\right) \geq \rho\right\}\right)^{*}=\left(\omega^{\leftarrow}(\rho)\right)^{*}
\end{aligned}
$$

We have $\xi_{\omega}^{\leftarrow} \in J(Y, X)$ from:

$$
\begin{aligned}
\bigvee_{i \in \Gamma} \xi_{\omega}^{\leftarrow}\left(\rho_{i}\right) \leq \lambda & \Leftrightarrow \xi_{\omega}^{\leftarrow}\left(\rho_{i}\right) \leq \lambda, \quad \forall i \in \Gamma \\
& \Leftrightarrow \omega \rightarrow\left(\lambda^{*}\right) \geq \rho_{i}, \quad \forall i \in \Gamma \\
& \Leftrightarrow \omega \rightarrow\left(\lambda^{*}\right) \geq \bigvee_{i \in \Gamma} \rho_{i}, \\
& \Leftrightarrow \xi_{\omega}^{\leftarrow}\left(\bigvee_{i \in \Gamma} \rho_{i}\right) \leq \lambda .
\end{aligned}
$$

$\omega^{\rightarrow}(\alpha \odot \lambda) \geq \alpha \rightarrow \omega^{\rightarrow}(\lambda)$ iff $\omega^{\leftarrow}(\alpha \odot \rho) \leq \alpha \rightarrow \omega^{\leftarrow}(\rho)$ iff $\left(\omega^{\leftarrow}(\alpha \odot \rho)\right)^{*} \geq\left(\alpha \rightarrow \omega^{\leftarrow}(\rho)\right)^{*}$ iff $\alpha \odot \xi_{\omega}^{\leftarrow}(\rho) \leq$ $\xi_{\omega}^{\leftarrow}(\alpha \odot \rho)$

(6) We have

$$
\begin{aligned}
\begin{aligned}
\xi_{\omega}(\lambda) & =\bigvee\left\{\rho \in L^{Y} \mid \xi_{\omega}^{\leftarrow}(\rho) \leq \lambda\right\} \\
& =\bigvee\left\{\rho \in L^{Y} \mid \omega \rightarrow\left(\lambda^{*}\right) \geq \rho\right\}=\omega \rightarrow\left(\lambda^{*}\right)
\end{aligned} \\
\xi_{\omega}(\alpha \rightarrow \lambda) \quad=\omega \rightarrow\left((\alpha \rightarrow \lambda)^{*}\right)=\omega \rightarrow\left(\alpha \odot \lambda^{*}\right) \\
\\
\quad \geq \alpha \rightarrow \omega \rightarrow\left(\lambda^{*}\right)=\alpha \rightarrow \xi_{\omega}(\lambda)
\end{aligned}
$$

$$
\begin{aligned}
\xi_{\omega}^{\leftarrow}\left(\xi_{\omega}\left(\xi_{\omega}^{\leftarrow}(\rho)\right)\right) & =\xi_{\omega}^{\leftarrow}\left(\xi_{\omega}\left(\left(\omega^{\leftarrow}(\rho)\right)^{*}\right)\right. \\
& =\xi_{\omega}^{\leftarrow}\left(\omega^{\rightarrow}\left(\omega^{\leftarrow}(\rho)\right)\right) \\
& =\left(\omega^{\leftarrow}\left(\omega^{\rightarrow}\left(\omega^{\leftarrow}(\rho)\right)\right)\right)^{*} \\
& =\left(\omega^{\leftarrow}(\rho)\right)^{*}=\xi_{\omega}^{\leftarrow}(\rho)
\end{aligned}
$$

Corollary 2.7. Let $P(X)$ and $P(Y)$ be families of subsets of $X$ and $Y$. Let $\omega \rightarrow: P(X) \rightarrow P(Y)$ be an operator with $\omega \rightarrow\left(\bigcup A_{i}\right)=\bigcap \omega \rightarrow\left(A_{i}\right)$ for $A_{i} \in P(X)$. Define functions $\phi_{\omega}, \xi_{\omega}: P(X) \rightarrow P(Y)$ and $\omega^{\leftarrow}, \phi_{\omega}^{\leftarrow}, \xi_{\omega}^{\leftarrow}$ : $P(Y) \rightarrow P(X)$ as follows: for all $A \in P(X), B \in P(Y)$,

$$
\begin{aligned}
& \omega^{\leftarrow}(B)=\bigcup\left\{A \in P(X) \mid \omega^{\rightarrow}(A) \supset B\right\} \\
& \phi_{\omega}^{\rightarrow}(A)=(\omega \rightarrow(A))^{c}, \phi_{\omega}^{\leftarrow}(B)=\omega^{\leftarrow}\left(B^{c}\right) \\
& \xi_{\omega}^{\leftarrow}(B)=\bigcap\left\{A \in P(X) \mid \omega\left(A^{c}\right) \geq B\right\}, \\
& \xi_{\omega}^{\rightarrow}(A)=\bigcup\left\{B \in P(Y) \mid \xi_{\omega}^{\leftarrow}(B) \subset A\right\}
\end{aligned}
$$

Then the following properties hold:

(1) $\omega^{\leftarrow}\left(\bigcup B_{i}\right)=\bigcap \omega^{\leftarrow}\left(B_{i}\right)$ for $B_{i} \in P(Y)$ with $\omega \rightarrow(A) \geq B \Leftrightarrow \omega^{\leftarrow}(B) \geq A$ for all $A \in P(X)$ and $B \in P(Y)$.

(2) The pair $\left(\omega^{\rightarrow}, \omega^{\leftarrow}\right)$ is an antitone Galois connection and $\left(\omega^{\leftarrow}\left(\omega^{\rightarrow}(A)\right), \omega \rightarrow(A)\right)$ for all $A \in P(X)$ are formal concepts.

(3) The pair $\left(\phi_{\omega}, \phi_{\omega}^{\leftarrow}\right)$ is an isotone Galois connection and $\left(\phi_{\omega}^{\leftarrow}\left(\phi_{\omega}(A)\right), \phi_{\omega}(A)\right)$ are attribute oriented concepts.

(4) $\xi_{\omega}^{\leftarrow}: P(Y) \rightarrow P(X)$ is a union-preserving function such that $\xi_{\omega}^{\leftarrow}(B)=\left(\omega^{\leftarrow}(B)\right)^{c}$ and

$$
\omega^{\rightarrow}\left(A^{c}\right) \supset B \Leftrightarrow \omega^{\leftarrow}(B) \supset A^{c} \Leftrightarrow \xi_{\omega}^{\leftarrow}(B) \subset A .
$$

(5) $\xi_{\omega}: P(X) \rightarrow P(Y)$ is an intersection-preserving function such that $\xi_{\omega}(A)=\omega^{\rightarrow}\left(A^{c}\right)$ and

$$
\omega^{\rightarrow}\left(A^{c}\right) \supset B \Leftrightarrow \omega^{\leftarrow}(B) \supset A^{c}
$$




$$
\Leftrightarrow \xi_{\omega}^{\leftarrow}(B) \subset A \Leftrightarrow B \subset \xi_{\omega}(A)
$$

(6) The pair $\left(\xi_{\omega}^{\leftarrow}, \xi_{\omega}\right)$ is an isotone Galois connection and $\left(\xi_{\omega}^{\leftarrow}(B), \xi_{\omega} \vec{\omega}\left(\xi_{\omega}^{\leftarrow}(B)\right)\right)$ for all $B \in P(Y)$ are object oriented concepts.

Example 2.8. Let $X=\left\{x_{1}, x_{2}, x_{3}\right\}, Y=\left\{y_{1}, y_{2}, y_{3}\right\}$ and $L=\{0,1\}$ be sets. Define a function $f: X \rightarrow Y$ as follows:

$$
f\left(x_{1}\right)=f\left(x_{2}\right)=y_{1}, f\left(x_{3}\right)=y_{2} .
$$

Define $\omega^{\rightarrow}: P(X) \rightarrow P(Y)$ as $\omega^{\rightarrow}(A)=\left\{y_{2}\right\} \cup(f(A))^{c}$;

$$
\begin{gathered}
\omega^{\rightarrow}(\emptyset)=Y, \omega^{\rightarrow}\left(\left\{x_{1}\right\}\right)=\omega^{\rightarrow}\left(\left\{x_{2}\right\}\right)=\left\{y_{2}, y_{3}\right\}, \\
\omega^{\rightarrow}\left(\left\{x_{3}\right\}\right)=Y, \omega \rightarrow\left(\left\{x_{1}, x_{2}\right\}\right)=\left\{y_{2}, y_{3}\right\}, \\
\omega^{\rightarrow}\left(\left\{x_{2}, x_{3}\right\}\right)=\omega^{\rightarrow}\left(\left\{x_{1}, x_{3}\right\}\right)=\omega^{\rightarrow}(X)=\left\{y_{2}, y_{3}\right\} . \\
\omega^{\leftarrow}(\emptyset)=\omega^{\leftarrow}\left(\left\{y_{2}\right\}\right)=\omega^{\leftarrow}\left(\left\{y_{2}, y_{3}\right\}\right)=\omega^{\leftarrow}\left(\left\{y_{3}\right\}\right)=X, \\
\omega^{\leftarrow}\left(\left\{y_{1}\right\}\right)=\omega^{\leftarrow}\left(\left\{y_{1}, y_{3}\right\}\right)=\left\{x_{3}\right\}, \\
\omega^{\leftarrow}\left(\left\{y_{1}, y_{2}\right\}\right)=\omega^{\leftarrow}(Y)=\left\{x_{3}\right\} .
\end{gathered}
$$

Thus, we obtain attribute oriented concepts $\left(\omega^{\leftarrow}\left(\omega^{\rightarrow}(\mu)\right), \omega \rightarrow(\mu)\right)$ as follows:

$$
\left\{\left(\left\{x_{3}\right\}, Y\right),\left(X,\left\{y_{2}, y_{3}\right\}\right)\right\}
$$

(2) $\phi_{\omega} \rightarrow(A)=\left\{y_{1}, y_{3}\right\} \cap f(A)$. Then

$$
\begin{aligned}
& \phi_{\vec{\omega}}(\emptyset)=\emptyset, \phi_{\vec{\omega}}\left(\left\{x_{1}\right\}\right)=\left\{y_{1}\right\}, \phi_{\vec{\omega}}\left(\left\{x_{2}\right\}\right)=\left\{y_{1}\right\}, \\
& \phi_{\omega} \vec{\omega}\left(\left\{x_{3}\right\}\right)=\emptyset, \phi_{\omega}\left(\left\{x_{1}, x_{2}\right\}\right)=\left\{y_{1}\right\}, \\
& \phi_{\omega}\left(\left\{x_{2}, x_{3}\right\}\right)=\phi_{\omega}\left(\left\{x_{1}, x_{3}\right\}\right)=\phi_{\omega}(X)=\left\{y_{1}\right\} . \\
& \phi_{\omega}^{\leftarrow}(Y)=\phi_{\omega}^{\leftarrow}\left(\left\{y_{1}, y_{3}\right\}\right)=X \\
& \phi_{\omega}^{\leftarrow}\left(\left\{y_{1}\right\}\right)=\phi_{\omega}^{\leftarrow}\left(\left\{y_{1}, y_{2}\right\}\right)=X,
\end{aligned}
$$

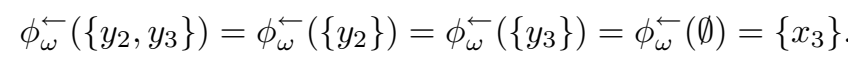

Thus, we obtain attribute oriented concepts $\left(\phi_{\omega}^{\overleftarrow{\omega}}\left(\phi_{\omega}(\mu)\right), \phi_{\omega}(\mu)\right)$ as follows:

$$
\left.\left\{\left(\left\{x_{3}\right\}, \emptyset\right\}\right),\left(X,\left\{y_{1}\right\}\right)\right\}
$$

(3) Since $\xi_{\omega} \rightarrow(A)=\omega \rightarrow\left(A^{c}\right), \xi_{\omega}^{\leftarrow}(B)=(\omega \rightarrow(B))^{c}$,

$$
\begin{gathered}
\xi_{\omega}(X)=Y, \xi_{\omega}\left(\left\{x_{2}, x_{3}\right\}\right)=\left\{y_{2}, y_{3}\right\}, \\
\xi_{\omega}\left(\left\{x_{1}, x_{3}\right\}\right)=\left\{y_{2}, y_{3}\right\}, \xi_{\omega}\left(\left\{x_{1}, x_{2}\right\}\right)=Y \\
\xi_{\omega}\left(\left\{x_{3}\right\}\right)=\left\{y_{2}, y_{3}\right\}, \\
\xi_{\omega}\left(\left\{x_{1}\right\}\right)=\xi_{\omega}\left(\left\{x_{2}\right\}\right)=\xi_{\omega}(\emptyset)=\left\{y_{2}, y_{3}\right\} . \\
\left.\xi_{\omega}^{\leftarrow} \emptyset\right)=\xi_{\omega}^{\leftarrow}\left(\left\{y_{2}\right\}\right)=\xi_{\omega}^{\leftarrow}\left(\left\{y_{2}, y_{3}\right\}\right)=\xi_{\omega}^{\leftarrow}\left(\left\{y_{3}\right\}\right)=\emptyset, \\
\xi_{\omega}^{\leftarrow}\left(\left\{y_{1}\right\}\right)=\xi_{\omega}^{\leftarrow}\left(\left\{y_{1}, y_{3}\right\}\right)=\left\{x_{1}, x_{2}\right\} . \\
\xi_{\omega}^{\leftarrow}\left(\left\{y_{1}, y_{2}\right\}\right)=\xi_{\omega}^{\leftarrow}(Y)=\left\{x_{1}, x_{2}\right\} .
\end{gathered}
$$

Thus, we obtain attribute oriented concepts $\left(\xi_{\omega}(\mu), \xi_{\omega}^{\leftarrow}\left(\xi_{\omega}(\mu)\right)\right)$ as follows:

$$
\left\{\left(\left\{x_{1}, x_{2}\right\}, Y\right),\left(\emptyset,\left\{y_{2}, y_{3}\right\}\right)\right\}
$$

Theorem 2.9. Let $(X, Y, R)$ be a fuzzy context. Define a function $\omega_{R}: L^{X} \rightarrow L^{Y}$ as follows:

$$
\omega_{R} \vec{R}(\lambda)(y)=\bigvee_{x \in X}(\lambda(x) \rightarrow R(x, y))
$$

Then we have the following properties:

(1) $\omega_{R} \in K(X, Y)$ and $\omega_{R}$ has a right adjoint mapping $\omega_{R}^{\leftarrow}$ with

$$
\omega_{R}^{\overleftarrow{R}}(\rho)(x)=\bigwedge_{y \in Y}(\rho(y) \rightarrow R(x, y))
$$

Moreover, $\omega_{R}^{\leftarrow}\left(\omega_{R}(\lambda)\right) \geq \lambda$ and $\omega_{R}\left(\omega_{R}^{\leftarrow}(\rho)\right) \geq \rho$ for all $\lambda \in L^{X}$ and $\rho \in L^{Y}$.

(2) $\left(\omega_{R}, \omega_{R}^{\leftarrow}\right)$ is an isotone Galois connections and $\left(\omega_{R}^{\overleftarrow{R}}\left(\omega_{R}(\lambda)\right), \omega_{R}(\lambda)\right)$ for all $\lambda \in L^{X}$ are formal concepts.

(3) $\omega_{R}(\alpha \odot \lambda)=\alpha \rightarrow \omega_{R}(\lambda)=\omega_{\alpha \rightarrow R}(\lambda)$ and $\omega_{R}^{\leftarrow}(\alpha \odot \rho)=\alpha \rightarrow \omega_{R}^{\leftarrow}(\rho)=\omega_{\alpha \rightarrow R}^{\leftarrow}(\rho)$, for all $\lambda \in$ $L^{X}, \rho \in L^{Y}$.

(4) $\phi_{\omega_{R}}(\mu)=\left(\omega_{R}(\mu)\right)^{*}$ and $\phi_{\omega_{R}}^{\leftarrow}(\rho)=\omega_{R}^{\overleftarrow{R}}\left(\rho^{*}\right)$ where

$$
\begin{aligned}
& \phi_{\omega_{R}}(\mu)(y)=\bigvee_{y \in Y}\left(\mu(x) \odot R^{*}(x, y)\right), \\
& \phi_{\omega_{R}}^{\leftarrow}(\rho)(x)=\bigwedge_{y \in Y}\left(R^{*}(x, y) \rightarrow \rho(y)\right) .
\end{aligned}
$$

(5) The pair $\left(\phi_{\omega_{R}}, \phi_{\omega_{R}}^{\leftarrow}\right)$ is an isotone Galois connection and $\left(\phi_{\omega_{R}}^{\leftarrow}\left(\phi_{\omega_{R}}(\lambda)\right), \phi_{\omega_{R}}(\lambda)\right)$ are attribute concepts.

(6)

$$
\begin{aligned}
& \xi_{\omega_{R}}^{\leftarrow}(\rho)(x)=\bigvee_{y \in Y}\left(\rho(y) \odot R^{*}(x, y)\right), \\
& \xi_{\omega_{R}}^{\rightarrow}(\lambda)(y)=\bigwedge_{x \in X}\left(R^{*}(x, y) \rightarrow \lambda(x)\right)
\end{aligned}
$$

(7) The pair $\left(\xi_{\omega_{R}}^{\leftarrow}, \xi_{\omega_{R}}\right)$ is an isotone Galois connection and $\left(\xi_{\omega_{R}}^{\leftarrow}(\rho), \xi_{\omega_{R}}\left(\xi_{\omega_{R}}^{\leftarrow}(\rho)\right)\right)$ for all $\rho \in L^{Y}$ are object oriented concepts. 
Proof. (1) Since $\omega_{R}\left(\bigvee_{i \in \Gamma} \lambda_{i}\right)(y)=\bigwedge_{x \in X}\left(\bigvee_{i \in \Gamma} \lambda_{i}(x) \rightarrow\right.$ $R(x, y))=\bigwedge_{i \in \Gamma}\left(\bigwedge_{x \in X}\left(\lambda_{i}(x) \rightarrow R(x, y)\right)=\right.$ $\bigwedge_{i \in \Gamma} \omega_{R}\left(\lambda_{i}\right)(y), \omega_{R}$ has a right adjoint mapping $\omega_{R}^{\leftarrow}$ as follows:

$$
\begin{aligned}
\omega_{R}^{\leftarrow}(\rho)(x) & =\bigvee\left\{\lambda \mid \rho \leq \omega_{R}(\lambda)\right\} \\
& =\bigvee\{\lambda \mid \rho(y) \leq \bigwedge(\lambda(x) \rightarrow R(x, y))\} \\
& =\bigvee\left\{\lambda \mid \lambda(x) \leq \bigwedge_{y \in Y}(\rho(y) \rightarrow R(x, y))\right\} \\
& =\bigwedge_{y \in Y}(\rho(y) \rightarrow R(x, y))
\end{aligned}
$$$$
\omega_{R}^{\leftarrow}\left(\omega_{R}(\lambda)\right)(x)
$$$$
=\bigwedge_{y \in Y}\left\{\omega_{R}(\lambda)(y) \rightarrow R(x, y)\right\}
$$$$
=\bigwedge_{y \in Y}\left\{\bigwedge_{x \in X}(\lambda(x) \rightarrow R(x, y)) \rightarrow R(x, y)\right\}
$$$$
\geq \bigwedge_{y \in Y}\{(\lambda(x) \rightarrow R(x, y)) \rightarrow R(x, y)\}
$$$$
\geq \lambda(x) \text {. }
$$$$
\omega_{R}\left(\omega_{R}^{\overleftarrow{R}}(\rho)\right)(y)
$$$$
=\bigwedge_{x \in X}\left\{\omega_{R}^{\leftarrow}(R)(\rho)(x) \rightarrow R(x, y)\right\}
$$$$
=\bigwedge_{x \in X}\left\{\bigwedge_{y \in Y}(\rho(y) \rightarrow R(x, y)) \rightarrow R(x, y)\right\}
$$$$
\geq \bigwedge_{x \in X}\{(\rho(y) \rightarrow R(x, y)) \rightarrow R(x, y)\}
$$$$
\geq \rho(y) \text {. }
$$

(3) By Lemma 1.3(6), we prove:

$$
\begin{aligned}
\omega_{R}(\alpha \odot \lambda)(y) & =\bigwedge_{x \in X}((\alpha \odot \lambda)(x) \rightarrow R(x, y)) \\
& =\bigwedge_{x \in X}(\alpha \rightarrow(\lambda(x) \rightarrow R(x, y))) \\
& =\alpha \rightarrow \bigwedge_{x \in X}(\lambda(x) \rightarrow R(x, y)) \\
& =\alpha \rightarrow \omega_{R}(\lambda)(y) \\
& =\bigwedge_{x \in X}(\lambda(x) \rightarrow(\alpha \rightarrow R(x, y))) \\
& =\omega_{\alpha \rightarrow R}^{\rightarrow}(\lambda)(y)
\end{aligned}
$$

(4)

$$
\begin{aligned}
& \phi_{\omega_{R}}(\mu)(y)=\left(\omega_{R}(\mu)\right)^{*}(y) \\
& =\left(\bigwedge_{x \in X}(\mu(x) \rightarrow R(x, y))^{*}\right. \\
& =\bigvee_{x \in X}\left(\mu(x) \odot R^{*}(x, y)\right)(\text { by Lemma 1.3(7)) } \\
& \phi_{\omega_{R}}^{\leftarrow}(\rho)(x)=\omega_{R}^{\leftarrow}\left(\rho^{*}\right)(x) \\
& =\bigvee_{y \in Y}\left(\rho^{*}(y) \rightarrow R(x, y)\right)(\text { by Lemma 1.3(7)) } \\
& =\bigwedge_{y \in Y}\left(R^{*}(x, y) \rightarrow \rho(y)\right)
\end{aligned}
$$

(5) It follows from Theorem 2.6(3).

(6)

$$
\begin{aligned}
\xi_{\omega_{R}}^{\leftarrow}(\rho)(x) & =\left(\omega_{R}^{\leftarrow}(\rho)(x)\right)^{*} \\
& =\left(\bigwedge_{y \in Y}(\rho(y) \rightarrow R(x, y))\right)^{*} \\
& =\bigvee_{y \in Y}\left(\rho(y) \odot R^{*}(x, y)\right) . \\
\xi_{\omega_{R}}(\mu)(y) & =\omega_{R}\left(\mu^{*}\right)(y) \\
& =\bigwedge_{x \in X}\left(\mu^{*}(x) \rightarrow R(x, y)\right) \\
& =\bigwedge_{x \in X}\left(R(x, y)^{*} \rightarrow \mu(x)\right) .
\end{aligned}
$$

(7) It follows from Theorem 2.6(7).
Corollary 2.10. Let $X$ and $Y$ be sets and $R \subset X \times Y$. Define a function $\omega_{R}: P(X) \rightarrow P(Y)$ as follows:

$$
\omega_{R}(A)=\{y \in Y \mid(\exists x \in X)(x \in A \rightarrow(x, y) \in R)\}
$$

Then we have the following properties:

(1) $\omega_{R} \in K(X, Y)$ and $\omega_{R}$ has a right adjoint mapping $\omega_{R}^{\leftarrow}$ with

$$
\omega_{R}^{\overleftarrow{ }}(B)=\{x \in X \mid(\exists y \in Y)(y \in B \rightarrow(x, y) \in R)\}
$$

Moreover, $\omega_{R}^{\leftarrow}\left(\omega_{R}(A)\right) \supset A$ and $\omega_{R}\left(\omega_{R}^{\leftarrow}(B)\right) \supset B$ for all $A \in P(X)$ and $B \in P(Y)$.

(2) $\left(\omega_{R}, \omega_{R}^{\leftarrow}\right)$ is an isotone Galois connections and $\left(\omega_{R}^{\leftarrow}\left(\omega_{R}(A)\right), \omega_{R}(A)\right)$ for all $A \in P(X)$ are formal concepts.

(3) $\phi_{\omega_{R}}(A)=\left(\omega_{R}(A)\right)^{c}$ and $\phi_{\omega_{R}}^{\leftarrow}(B)=\omega_{R}^{\leftarrow}\left(B^{c}\right)$ where

$\phi_{\omega_{R}}(A)=\left\{y \in Y \mid(\exists x \in A)\left((x \in A) \wedge\left((x, y) \in R^{c}\right)\right)\right\}$, $\phi_{\omega_{R}}^{\leftarrow}(B)=\left\{x \in X \mid(\forall y \in Y)\left((x, y) \in R^{c} \rightarrow y \in B\right)\right\}$.

(5) The pair $\left(\phi_{\omega_{R}}, \phi_{\omega_{R}}^{\leftarrow}\right)$ is an isotone Galois connection and $\left(\phi_{\omega_{R}}^{\leftarrow}\left(\phi_{\omega_{R}}(A)\right), \phi_{\omega_{R}}(A)\right)$ are attribute concepts.

(6)

$\xi_{\omega_{R}}^{\leftarrow}(B)=\left\{x \in X \mid(\exists y \in B)\left((y \in B) \wedge\left((x, y) \in R^{c}\right)\right\}\right.$,

$$
\xi_{\omega_{R}}(A)=\left\{y \in Y \mid(\forall x \in X)\left((x, y) \in R^{c} \rightarrow x \in A\right)\right\}
$$

(7) The pair $\left(\xi_{\omega_{R}}^{\leftarrow}, \xi_{\omega_{R}}\right)$ is an isotone Galois connection and $\left(\xi_{\omega_{R}}^{\leftarrow}(B), \xi_{\omega_{R}}\left(\xi_{\omega_{R}}^{\leftarrow}(B)\right)\right)$ for all $B \in P(Y)$ are object oriented concepts.

Example 2.11. Let $X=\{a, b, c\}, Y=\{x, y, z, w\}$ and $L=\{0,1\}$ be sets. Define a relation $R$ as follows:

$$
R=\{(a, x),(a, y),(b, y),(b, w),(c, z),(c, w)\} .
$$

(1) Define $\omega_{R}: P(X) \rightarrow P(Y)$ as $\omega_{R}(A)=\{y \in Y \mid$ $a \in A \rightarrow(a, y) \in R\}$;

$$
\begin{gathered}
\omega_{R} \overrightarrow{(\emptyset)}=Y, \omega_{R} \overrightarrow{(}(\{a\})=\{x, y\}, \omega_{R} \overrightarrow{ }(\{b\})=\{y, w\}, \\
\omega_{R}(\{c\})=\{z, w\}, \omega_{R}(\{a, b\})=\{y\}, \\
\omega_{\vec{R}}(\{b, c\})=\{w\}, \omega_{\vec{R}}(\{a, c\})=\omega_{\vec{R}}(X)=\emptyset .
\end{gathered}
$$

We obtain $\omega_{R}^{\overleftarrow{R}}(B)=\{a \in X \mid y \in B \rightarrow(a, y) \in R\}$;

$$
\begin{aligned}
& \omega_{R}^{\leftarrow}(\emptyset)=X, \omega_{R}^{\leftarrow}(\{w\})=\{b, c\} \\
& \omega_{R}^{\leftarrow}(\{z\})=\{c\}=\omega_{R}^{\leftarrow}(\{z, w\}), \\
& \omega_{R}^{\leftarrow}(\{y\})=\{a, b\}, \omega_{R}^{\leftarrow}(\{y, w\})=\{b\}=\omega_{R}^{\leftarrow}(\{x, y\}) \\
& \omega_{R}^{\leftarrow}(\{x\})=\{a\}, \omega_{R}^{\leftarrow}(\{y, z\})=\omega_{R}^{\overleftarrow{ }}(\{y, z, w\})=\emptyset, \\
& \omega_{R}^{\overleftarrow{R}}(\{x, w\})=\omega_{R}^{\leftarrow}(\{x, z\})=\omega_{R}^{\leftarrow}(\{x, z, w\})=\emptyset, \\
& \omega_{R}^{\leftarrow}(\{x, y, w\})=\omega_{R}^{\leftarrow}(\{x, y, z\})=\omega_{R}^{\leftarrow}(Y)=\emptyset,
\end{aligned}
$$


Thus, we obtain formal concepts

$$
\begin{gathered}
\{(\emptyset, Y),(\{a\},\{x, y\}),(\{b\},\{y, w\}),(\{c\},\{z, w\}) \\
(\{b, c\},\{w\}),(\{a, b\},\{y\})(X, \emptyset)\}
\end{gathered}
$$

(2) We obtain a relation $R^{*}=R^{c}$ as follows:

$$
R^{c}=\{(a, z),(a, w),(b, x),(b, z),(c, x),(c, y)\} .
$$

We obtain $\phi_{\omega_{R}}: P(X) \rightarrow P(Y)$ as

$$
\begin{gathered}
\phi_{\omega_{R}}(A)=\left\{y \in Y \mid(\exists a \in A)\left(a \in A \wedge(a, y) \in R^{c}\right)\right\} \\
\phi_{\omega_{R}}(\emptyset)=\emptyset, \phi_{\omega_{R}}(\{a\})=\{z, w\}, \phi_{\omega_{R}}(\{b\})=\{x, z\}, \\
\phi_{\overrightarrow{\omega_{R}}}(\{c\})=\{x, y\} \phi_{\omega_{R}}(\{a, b\})=\{x, z, w\}, \\
\phi_{\omega_{R}}(\{b, c\})=\{x, y, z\}, \phi_{\omega_{R}}(\{a, c\})=\phi_{\omega_{R}}(X)=Y .
\end{gathered}
$$

We obtain $\phi_{\omega_{R}}^{\leftarrow}(B)=\{a \in X \mid y \in B \rightarrow(a, y) \in R\}$;

$$
\begin{gathered}
\phi_{\omega_{R}}^{\leftarrow}(\emptyset)=\phi_{\omega_{R}}^{\leftarrow}(\{x\})=\phi_{\omega_{R}}^{\leftarrow}(\{y\})=\phi_{\omega_{R}}^{\leftarrow}(\{z\})=\emptyset, \\
\phi_{\omega_{R}}^{\leftarrow}(\{w\})=\phi_{\omega_{R}}^{\leftarrow}(\{x, w\})=\emptyset, \\
\phi_{\omega_{R}}^{\leftarrow}(\{y, z\})=\phi_{\omega_{R}}^{\leftarrow}(\{y, w\})=\emptyset, \\
\phi_{\omega_{R}}^{\leftarrow}(\{x, y\})=\phi_{\omega_{R}}^{\leftarrow}(\{x, y, w\})=\{c\}, \phi_{\omega_{R}}^{\leftarrow}(\{z, w\})=\{b\}, \\
\phi_{\omega_{R}}^{\leftarrow}(Y)=X, \phi_{\omega_{R}}^{\leftarrow}(\{x, z\})=\{b\}, \phi_{\omega_{R}}^{\leftarrow}(\{y, z, w\})=\{a\}, \\
\phi_{\omega_{R}}^{\leftarrow}(\{x, z, w\})=\{a, b\}, \phi_{\omega_{R}}^{\leftarrow}(\{x, y, z\})=\{b, c\} .
\end{gathered}
$$

Thus, we obtain attribute oriented concepts

$$
\begin{gathered}
\{(\emptyset, \emptyset),(\{c\},\{x, y\}),(\{b\},\{x, z\}),(\{a\},\{z, w\}), \\
(\{b, c\},\{x, y, z\}),(\{a, b\},\{x, z, w\})(X, Y)\}
\end{gathered}
$$

(3) We obtain $\xi_{\omega_{R}}^{\leftarrow}(B)=\{a \in X \mid(\exists y \in B)((y \in$ $\left.\left.B) \wedge\left((a, y) \in R^{c}\right)\right)\right\}$;

$$
\begin{gathered}
\xi_{\omega_{R}}^{\leftarrow}(\emptyset)=\emptyset, \xi_{\omega_{R}}^{\leftarrow}(\{w\})=\{a\}, \xi_{\omega_{R}}^{\leftarrow}(\{y\})=\{c\}, \\
\xi_{\omega_{R}}^{\leftarrow}(\{z\})=\xi_{\omega_{R}}^{\leftarrow}(\{z, w\})=\{a, b\}, \xi_{\omega_{R}}^{\leftarrow}(\{y, w\})=\{a, c\}, \\
\xi_{\omega_{R}}^{\leftarrow}(\{x\})=\xi_{\omega_{R}}^{\leftarrow}(\{x, y\})=\{b, c\}, \xi_{\omega_{R}}^{\leftarrow}(\{y, z\})=X \\
\xi_{\omega_{R}}^{\leftarrow}(\{y, z, w\})=\xi_{\omega_{R}}^{\leftarrow}(\{x, w\})=\xi_{\omega_{R}}^{\leftarrow}(\{x, z\})=X, \\
\xi_{\omega_{R}}^{\leftarrow}(\{x, z, w\})=\xi_{\omega_{R}}^{\leftarrow}(\{x, y, w\})=X, \\
\xi_{\omega_{R}}^{\leftarrow}(\{x, y, z\})=\xi_{\omega_{R}}^{\leftarrow}(Y)=X . \\
\xi_{\omega_{R}}(A)=\left\{y \in Y \mid\left((a, y) \in R^{c} \rightarrow(a \in A)\right\} ;\right. \\
\xi_{\omega_{R}}(\emptyset)=\xi_{\omega_{R}}(\{b\})=\emptyset, \xi_{\omega_{R}}(\{c\})=\{y\}, \\
\xi_{\omega_{R}}(\{b, c\})=\{x, y\}, \xi_{\omega_{R}}(\{a\})=\{w\}, \\
\xi_{\omega_{R}}(\{b, c\})=\{x, y\}, \xi_{\omega_{R}}(\{a, c\})=\{y, w\}, \xi_{\omega_{R}}(X)=Y .
\end{gathered}
$$

Thus, we obtain object oriented concepts

$$
\begin{gathered}
\{(\emptyset, \emptyset),(\{c\},\{y\}),(\{b, c\},\{x, y\}),(\{a\},\{w\}) \\
(\{a, c\},\{y, w\}),(\{a, b\},\{z, w\}),(X, Y)\}
\end{gathered}
$$

\section{References}

[1] R. Bělohlávek," Similarity relations in concept lattices," J. Logic and Computation, vol. 10, no. 6, pp. 823-845, 2000.

[2] R. Bělohlávek, "Lattices of fixed points of Galois connections," Math. Logic Quart., vol. 47, pp.111-116, 2001.

[3] R. Bělohlávek," Concept lattices and order in fuzzy logic," Ann. Pure Appl. Logic, vol. 128, pp. 277298, 2004.

[4] R. Bělohlávek, Fuzzy relational systems, Kluwer Academic Publisher, New York, 2002.

[5] G. Georgescu, A. Popescue, "Non-dual fuzzy connections," Arch. Math. Log., vol. 43, pp.1009-1039, 2004.

[6] U. Höhle, E. P. Klement, Non-classical logic and their applications to fuzzy subsets, Kluwer Academic Publisher, Boston, 1995.

[7] Y.C. Kim, J.W. Park, "Join preserving maps and various concepts," Int.J. Contemp. Math. Sciences, vol.5, no.5, pp. 243-251, 2010.

[8] H. Lai, D. Zhang, "Concept lattices of fuzzy contexts: Formal concept analysis vs. rough set theory," Int. J. Approx. Reasoning, vol. 50, pp.695-707, 2009.

[9] E. Turunen, Mathematics Behind Fuzzy Logic, A Springer-Verlag Co., 1999.

[10] R. Wille,Restructuring lattice theory; an approach based on hierarchies of concept, in: 1. Rival(Ed.), Ordered Sets, Residel, Dordrecht, Boston, 1982.

\section{Jung Mi Ko}

She received the M.S and Ph.D. degrees in Department of Mathematics from Yonsei University, in 1983 and 1988, respectively. From 1988 to present, she is a professor in Department of Mathematics, Gangneung-Wonju University. Her research interests are fuzzy logic.

\section{Yong Chan Kim}

He received the M.S and Ph.D. degrees in Department of Mathematics from Yonsei University, in 1984 and 1991, respectively. From 1991 to present, he is a professor in Department of Mathematics, Gangneung-Wonju University. His research interests are fuzzy logic and fuzzy topology. 